\title{
Relativistic outflow from two thermonuclear shell flashes on neutron stars
}

\author{
J. J. M. in 't Zand ${ }^{1}$, L. Keek ${ }^{2}$, and Y. Cavecchi ${ }^{3}$ \\ ${ }^{1}$ SRON Netherlands Institute for Space Research, Sorbonnelaan 2, 3584 CA Utrecht, The Netherlands \\ e-mail: jeanz@sron.nl \\ 2 Center for Relativistic Astrophysics, School of Physics, Georgia Institute of Technology, Atlanta, USA \\ 3 Astronomical Institute "Anton Pannekoek", University of Amsterdam, Science Park 904, 1098 XH Amsterdam, The Netherlands
}

Received 22 April 2014 / Accepted 14 June 2014

\begin{abstract}
We study the exceptionally short (32-43 ms) precursors of two intermediate-duration thermonuclear X-ray bursts observed with the Rossi X-ray Timing Explorer from the neutron stars in 4U 0614+09 and 2S 0918-549. They exhibit photon fluxes that surpass those at the Eddington limit later in the burst by factors of 2.6 to 3.1. We are able to explain both the short duration and the super-Eddington flux by mildly relativistic outflow velocities of $0.1 c$ to $0.3 c$ subsequent to the thermonuclear shell flashes on the neutron stars. These are the highest velocities ever measured from any thermonuclear flash. The precursor rise times are also exceptionally short: about $1 \mathrm{~ms}$. This is inconsistent with predictions for nuclear flames spreading laterally as deflagrations and suggests detonations instead. This is the first time that a detonation is suggested for such a shallow ignition column depth $\left(y_{\mathrm{ign}} \approx 10^{10} \mathrm{~g} \mathrm{~cm}^{-2}\right)$. The detonation would possibly require a faster nuclear reaction chain, such as bypassing the $\alpha$-capture on ${ }^{12} \mathrm{C}$ with the much faster ${ }^{12} \mathrm{C}(p, \gamma){ }^{13} \mathrm{~N}(\alpha, p){ }^{16} \mathrm{O}$ process previously proposed. We confirm the possibility of a detonation, albeit only in the radial direction, through the simulation of the nuclear burning with a large nuclear network and at the appropriate ignition depth, although it remains to be seen whether the Zel'dovich criterion is met. A detonation would also provide the fast flame spreading over the surface of the neutron star to allow for the short rise times. This needs to be supported by future two-dimensional calculations of flame spreading at the relevant column depth. As an alternative to the detonation scenario, we speculate on the possibility that the whole neutron star surface burns almost instantly in the auto-ignition regime. This is motivated by the presence of $150 \mathrm{~ms}$ precursors with $30 \mathrm{~ms}$ rise times in some superexpansion bursts from $4 \mathrm{U} 1820-30$ at low ignition column depths of $\sim 10^{8} \mathrm{~g} \mathrm{~cm}^{-2}$.
\end{abstract}

Key words. X-rays: binaries - X-rays: individuals: 4U 0614+09 - X-rays: individuals: 2S 0918-549 - X-rays: bursts - stars: neutron

\section{Introduction}

Thermonuclear shell flashes on neutron stars (NSs) heat up the photospheres to typical temperatures of $10^{7} \mathrm{~K}$, giving rise to Type I X-ray bursts (Grindlay et al. 1976; Woosley \& Taam 1976; Maraschi \& Cavaliere 1977; Joss 1977; Swank et al. 1977; Lamb \& Lamb 1978; Lewin et al. 1993; Strohmayer \& Bildsten 2006). The primary fuel for such flashes (hydrogen and helium) is provided by the companion star in the hosting low-mass X-ray binary (LMXB). Currently, about 100 bursting NSs are known in our galaxy, providing a galactic flash rate of a few per hour. Luminosities often reach $\sim 10^{38} \mathrm{erg} \mathrm{s}^{-1}$, making X-ray bursts easily detectable throughout the galaxy.

In the past two decades, the harvest of X-ray bursts was rich. This is due to the Proportional Counter Array (PCA) on RXTE (1995-2012; e.g., Jahoda et al. 2006; Galloway et al. 2008), the Wide Field Cameras on BeppoSAX (1996-2002; e.g., Jager et al. 1997; in't Zand et al. 2004) and JEM-X on INTEGRAL (launched in 2003 and still active; e.g., Lund et al. 2003), each yielding at least 2100 burst detections ${ }^{1}$. The instrument with the largest photon collecting area is the PCA, at about $8000 \mathrm{~cm}^{2}$, compared to a few hundred $\mathrm{cm}^{2}$ for the other two instruments. This yields, for a few-keV blackbody spectrum shining at the Eddington limit at a distance equal to that of the galactic center,

\footnotetext{
1 These are collected in the MINBAR database (Galloway et al. 2010), see http://burst. sci.monash.edu/minbar
}

typical photon rates of $10^{4} \mathrm{~s}^{-1}$. The PCA data, therefore, are particularly well suited to the study of X-ray bursts at millisecond timescale.

Most of the X-ray burst signal is due to cooling of the burned layer. The cooling time, or burst duration, scales with the amount of cooling matter, therefore with the thickness of the layer (e.g., in't Zand et al. 2014). Most bursts ignite at a column depth of $y_{\text {ign }} \sim 10^{8} \mathrm{~g} \mathrm{~cm}^{-2}$ and have durations of $\sim 1 \mathrm{~min}$. At the other end of the spectrum are the so-called superbursts with ignition column depths of $y_{\text {ign }} \sim 10^{11-12} \mathrm{~g} \mathrm{~cm}^{-2}$ and durations of $\sim 1 \mathrm{~d}$ (Cornelisse et al. 2000; Cumming \& Bildsten 2001; Strohmayer \& Brown 2002; Keek \& in't Zand 2008). Bursts that last $\sim 1 \mathrm{~h}$ are called intermediate duration bursts and have intermediate ignition column depths of $y_{\text {ign }} \sim 10^{10} \mathrm{~g} \mathrm{~cm}^{-2}$ (in't Zand et al. 2005; Cumming et al. 2006). These are of special interest here. They are thought to arise when there is a relatively thick pile of helium on a relatively cool NS. On a cold NS, the ignition temperature is reached deeper in the envelope, ergo the thick ignition layer. Such ignition conditions are readily found (in't Zand et al. 2007) in ultracompact X-ray binaries (UCXBs), in which the NS is accompanied by a hydrogen-poor helium-rich white dwarf in a compact orbit of period less than $\sim 1 \mathrm{~h}$ (e.g., Nelson et al. 1986). The white dwarf is thought to be the hydrogen-poor core of a star denuded in the past of its hydrogen-rich atmosphere by the accretion process. Ignition of thick helium piles on cool NSs will provide the highest nuclear power. 
The luminosity during an X-ray burst is determined by the fuel amount and composition, which dictates specific reaction rates through the associated nuclear reaction chain. In $20 \%$ of the bursts (Galloway et al. 2008) the luminosity is high enough that it reaches the Eddington limit. This happens particularly when the energy production is dominated by helium burning via the $3 \alpha$ process and subsequent $\alpha$-captures. Although helium burning yields only roughly $1 / 3$ of the energy per nucleon that hydrogen burning does, the reaction rate overcompensates this to an extent that the nuclear power from $3 \alpha$ burning is higher than from CNO hydrogen burning (Fujimoto et al. 1981; Bildsten 1998). When the power reaches the Eddington limit (i.e., most nuclear power transforms to radiation, except possibly during superbursts, see Cumming et al. 2006), the NS photosphere expands due to radiation pressure. The photosphere will, at the same time, cool adiabatically (e.g., Grindlay et al. 1980). When the expansion is large enough (we call this superexpansion; in't Zand \& Weinberg 2010), the temperature will move out of the bandpass and the X-ray signal is lost (Tawara et al. 1984a,b; Lewin et al. 1984). The signal returns when the photosphere moves back to the NS and the superexpansion phase is over. One is left with the appearance of a precursor. If intermediate duration bursts are due to helium burning, a high luminosity and superexpansion is particularly expected since the fuel amount is large and the nuclear reaction rate high.

There are two hypotheses about the nature of the expanded photosphere in superexpansion bursts. Both attribute the effect to an expansion to at least $10^{3} \mathrm{~km}$, in line with the observation (e.g., Molkov et al. 2000) that not only the burst radiation fades, but also the accretion radiation since the X-ray emitting portion of the accretion disk is covered by the expanded NS photosphere. The first hypothesis (argued by, e.g., Wallace et al. 1982; Ebisuzaki et al. 1983; Paczynski \& Proszynski 1986; Joss \& Melia 1987; Nobili et al. 1994) entails a steady-state lowvelocity $(\$ 0.01 c)$ wind. in't Zand \& Weinberg (2010) noted two problems with this model. First, it does not explain why the photosphere returns to the NS surface fairly quickly, while the luminosity does not change as fast and is still Eddington limited. Second, it does not explain why the superexpansion duration is independent of burst duration, equivalent to ignition depth. Therefore, in't Zand \& Weinberg (2010) propose that something important happens during the initial stage of the burst, when the quasi-static wind has not established yet: the expulsion of a shell, like in a nova, which expands and cools whereby the radiation moves below the bandpass. The shell keeps expanding during the dark stage, being driven by continued Eddington-limited radiation pressure. It dilutes and, at the end of the superexpansion stage, becomes optically thin. At this point the NS underneath shines through it, still radiating at the Eddington limit. The apparent radius is a mix of the far-away shell, which scatters the NS emission, and the NS and drops on a timescale of a few seconds. The NS photosphere still radiates at the Eddington limit and is slightly expanded. This is likely the quasi-static wind argued by, e.g., Paczynski \& Proszynski (1986) and Joss \& Melia (1987). It is less expanded than predicted $\left(\sim 10^{1-2} \mathrm{~km}\right.$ instead of $10^{2-3} \mathrm{~km}$ ), possibly because those predictions do not take into account line driving in a recombined gas expelling the upper cooler parts of the wind (see in't Zand \& Weinberg 2010, for a more detailed discussion). Joss \& Melia (1987) show that it takes about $1 \mathrm{~s}$ for a wind to reach a static state. It may be during this dynamic stage that the geometrically thin optically thick shell is expelled, according to in't Zand \& Weinberg (2010). Interestingly, for a temperature of $\gtrsim 1 \mathrm{GK}$, the opacity becomes smaller because it is dominated by Compton instead of
Table 1. Basic parameters of the two bursts (some information from Kuulkers et al. 2010 and in't Zand et al. 2011).

\begin{tabular}{|c|c|c|}
\hline Parameter & 4U 0614+09 & 2S 0918-549 \\
\hline Time (MJD) & 51944.91157 & 54504.12698 \\
\hline Date & 2001-Feb.-04 & 2008-Feb.-08 \\
\hline RXTE ObsID & 50031-01-03-05 & $93416-01-05-00$ \\
\hline PCUs & $0,1,2,3$ & 0,2 \\
\hline Fluence $\left(\mathrm{erg} \mathrm{cm}^{-2}\right.$ ) & $3.17 \times 10^{-5}$ & $1.90 \times 10^{-5}$ \\
\hline Rad. energy outp. (erg) & $3.4 \times 10^{40}$ & $7 \times 10^{40}$ \\
\hline Peak flux (erg $\left.\mathrm{cm}^{-2} \mathrm{~s}^{-1}\right)$ & $2.66 \times 10^{-7}$ & $1.18 \times 10^{-7}$ \\
\hline Timescale $(\mathrm{s})^{a}$ & 120 & 160 \\
\hline Precursor duration ${ }^{b}$ (ms) & $43 \pm 1$ & $32 \pm 1$ \\
\hline Superexpansion duration (s) & $1.15 \pm 0.01$ & $1.25 \pm 0.01$ \\
\hline Touch-down point (s) & $75 \pm 5$ & $75 \pm 5$ \\
\hline Ignition column depth $\left(\mathrm{g} \mathrm{cm}^{-2}\right)$ & $8 \times 10^{9}$ & $(1-2) \times 10^{10}$ \\
\hline Bol. pers. flux $\left(\mathrm{erg} \mathrm{cm}^{-2} \mathrm{~s}^{-1}\right)$ & $\begin{array}{l}3.77 \times 10^{-9} \\
(1.4 \% \text { of peak flux })\end{array}$ & $\begin{array}{l}5.5 \times 10^{-10} \\
(0.5 \%)\end{array}$ \\
\hline
\end{tabular}

Notes. ${ }^{(a)}$ The timescale is defined as the fluence divided by peak flux (see Galloway et al. 2008); ${ }^{(b)}$ The precursor duration is measured between the times that the precursor rises above and decays below $5 \%$ of the peak flux in the full PCA bandpass.

Thompson scattering. As a result, the Eddington limit increases going into the flash layer by a factor of up to five (Hanawa \& Sugimoto 1982). Therefore, at the start of a burst, a larger fraction of the layer may be expelled if the nuclear luminosity is larger. The column thickness of the expelled shell should be at least $\sim 10^{4} \mathrm{~g} \mathrm{~cm}^{-2}$ to remain optically thick $\left(>1 \mathrm{~g} \mathrm{~cm}^{-2}\right)$ up to a distance of $\sim 10^{3} \mathrm{~km}$. On the other hand, it cannot be thicker than about $1 \%$ of the ignition column depth, because the nuclear burning does not provide enough energy (between $1.6 \mathrm{MeV}$ and 4.4 MeV per nucleon; Fujimoto et al. 1987) to transport more mass out of the NS gravitational well $(\approx 200 \mathrm{MeV}$ per nucleon for a canonical NS with mass $1.4 M_{\odot}$ and radius $10 \mathrm{~km}$ ).

Thirty-nine superexpansion bursts have been detected from 9 sources throughout the 50-year history of X-ray astronomy (see Appendix A). More than half are from a single source (4U 1722-30). In almost all cases, precursors last about $1 \mathrm{~s}$. Two intermediate duration bursts detected with the PCA from 4U 0614+09 and 2S 0918-549 form an exception, with precursors lasting a mere $32-43 \mathrm{~ms}$. These two bursts are the subject of our study. The fast precursors immediately point to very fast shell velocities and provide interesting constraints on the physics of ignition, nuclear burning, flame spreading, and dynamical phenomena of the NS photosphere. In Sect. 2 we introduce the general properties of the two bursts, citing results of previous studies. Section 3 reports the analysis of the timing and spectral properties of the two precursors. In Sect. 4 we interpret the properties in terms of the physical aspects mentioned above. We conclude in Sect. 5 and discuss future prospects.

\section{Introducing the two bursts}

The initial phases of both bursts were detected with the PCA. The PCA consists of five co-aligned proportional counter units (PCUs) that combine to a $8000 \mathrm{~cm}^{2}$ peak effective area at $6 \mathrm{keV}$ in a 2-60 keV bandpass (Jahoda et al. 2006). The spectral resolution is about $20 \%$ (full width at half maximum) at best and the mostly used data collecting mode, including here, allows a time resolution of $122 \mu \mathrm{s}$. Generally, not all PCUs operate at the same time. The burst light curves are shown in Fig. 1. Derived parameters are listed in Table 1. Here follows a summary of the earlier findings. 

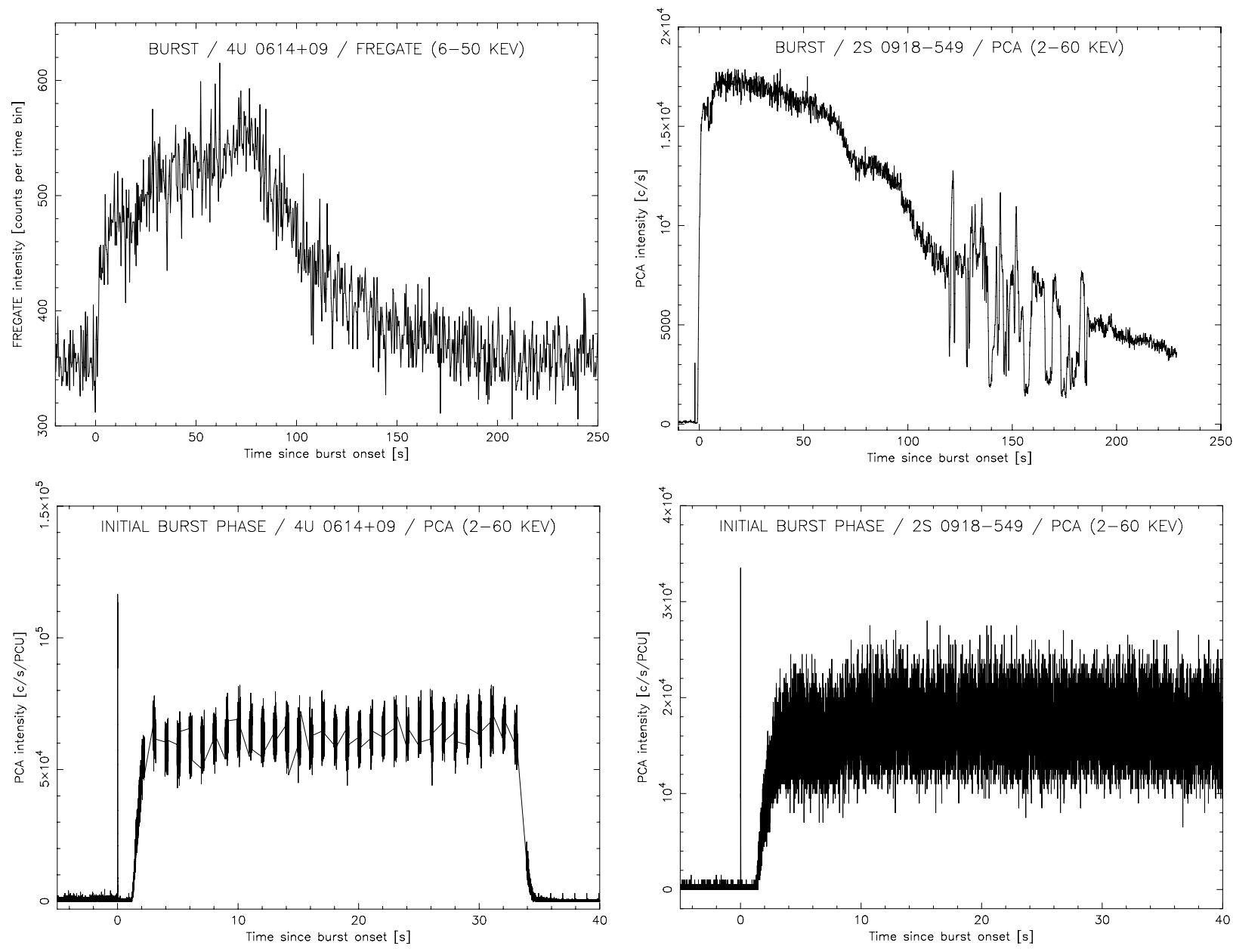

Fig. 1. Top panels: light curves of the major parts of the bursts, left of the burst from 4U 0614+09 as measured in 6-50 keV with HETE-IIFREGATE (Kuulkers et al. 2010) and right from 2S 0918-549 as measured in 2-60 keV with the PCA (in't Zand et al. 2011). In both bursts, the end of the photospheric expansion phase (the so-called touch-down point) occurs at $75 \mathrm{~s}$, recognizable as a peak in the 6-50 keV flux for 4U 0614+09 and as a dip in the 2-60 keV flux for $2 \mathrm{~S}$ 0918-549. Bottom panels: zoomed in light curves of the same bursts at 2 ms resolution, as measured with the PCA. These high-time-resolution measurements for 4U 0614+09 suffer from regular data gaps due to telemetry saturation. The drop at the end of $4 \mathrm{U} 0614+09$ is due to the PCA being shut off after the high count rate safety threshold was exceeded. No dead time corrections were applied.

The source $4 \mathrm{U} 0614+09$ contains a NS that has been accreting for at least $40 \mathrm{yr}$ (Giacconi et al. 1974). It is an UCXB with an orbital period of probably 50 min (Shahbaz et al. 2008). There are negligible amounts of hydrogen being accreted by the NS, as shown by optical spectroscopy (Nelemans et al. 2006). Kuulkers et al. (2010) investigated the burst activity since its discovery and found 30 bursts in almost 40 years of data, with very bright peak fluxes of up to 15 times the Crab source. Apart from the record holder Cen X-4 (Belian et al. 1972; Kuulkers et al. 2009), this is the brightest of all bursters which makes it an excellent target for studies at small timescales. The distance to $4 \mathrm{U} 0614+09$ has been determined at $3.2 \mathrm{kpc}$ from equating the peak flux of Eddington-limited bursts to the Eddington luminosity limit expected for a hydrogen-poor atmosphere (Brandt et al. 1992; Kuulkers et al. 2010). The average accretion rate is low at only $0.8 \%$ of the H-poor Eddington limit (in't Zand et al. 2007). The burst we investigate here is discussed in Kuulkers et al. (2010) and in in't Zand \& Weinberg (2010). It is the brightest burst detected with RXTE. Kuulkers et al. determined an ignition column depth of $y_{\mathrm{ign}}=8 \times 10^{9} \mathrm{~g} \mathrm{~cm}^{-2}$ by modeling the tail of the burst with a cooling envelope. The burst is only partly covered by PCA observations, as the observation ended $33 \mathrm{~s}$ after burst onset. The remainder of the burst was measured with other instruments and, after $1.2 \mathrm{~h}$, again with the PCA. The duration is long, with an estimated $6-50 \mathrm{keV}$ e-folding decay time of $40 \mathrm{~s}$. It is a typical intermediate duration burst (e.g., in't Zand et al. 2005; Cumming et al. 2006).

The source $2 \mathrm{~S} 0918-549$ is similar to $4 \mathrm{U} 0614+09$, but at a larger distance (5.4 kpc; Nelemans et al. 2004; in't Zand et al. 2005). The tentatively measured orbital period is $17 \mathrm{~min}$ (Zhong \& Wang 2011) which would also make it an ultracompact X-ray binary, as already suspected on the basis of optical spectroscopy (Nelemans et al. 2004). The average accretion rate is $0.5 \%$ of the Eddington limit (in't Zand et al. 2007). The burst we discuss was published previously in in't Zand et al. (2011). The fluence translates to an energy output of $7 \times 10^{40} \mathrm{erg}$ for a distance of $5.4 \mathrm{kpc}$. We estimate, on the basis of the same method as applied on the burst of 4U 0614+09 (see above and Kuulkers et al. 2010), that the ignition column depth is $y_{\mathrm{ign}}=(1-2) \times 10^{10} \mathrm{~g} \mathrm{~cm}^{-2}$ (A. Cumming, priv. comm.). The duration is longer than $310 \mathrm{~s}$, at which time the observation ended. Therefore, this is a clear intermediate duration burst from the prototypical source of such bursts (in't Zand et al. 2005). Later on in the burst (120 s to $190 \mathrm{~s}$ after burst onset; see Fig. 1) strong upward and downward modulations occur, which are explained by the effects of an accretion disk which was dynamically disturbed by the burst outflow and 

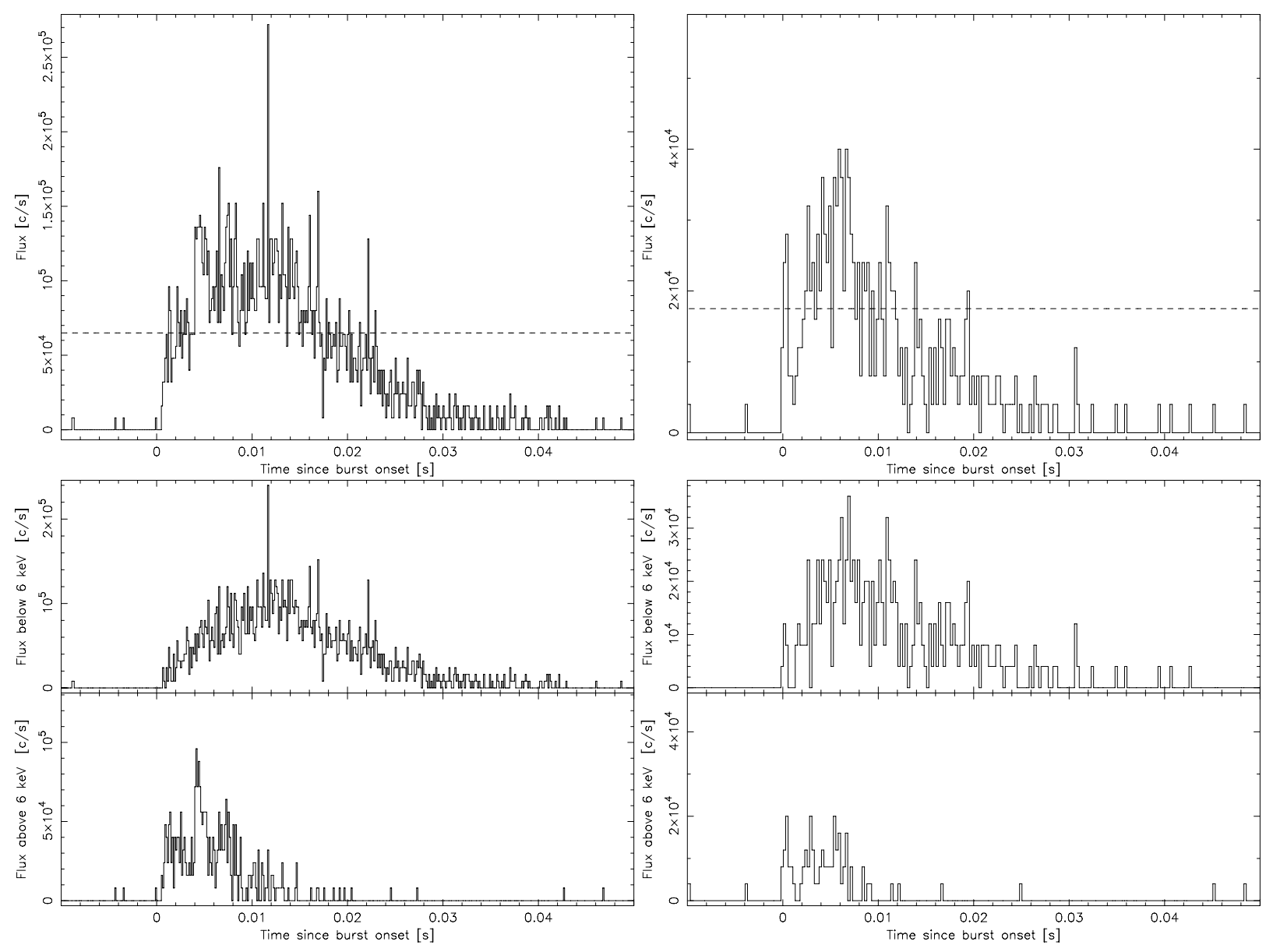

Fig. 2. X-ray light curves of the precursor to the burst from $4 \mathrm{U} 0614+09$ at $122 \mu \mathrm{s} \mathrm{time} \mathrm{resolution} \mathrm{(left} \mathrm{panels)} \mathrm{and} \mathrm{the} \mathrm{burst} \mathrm{from} 2 \mathrm{~S} 0918-549$ at $0.25 \mathrm{~ms}$ resolution (right panels). The top graphs refers to photons at all photon energies, the middle ones to photons of energies below $6 \mathrm{keV}$, and the bottom ones to those above $6 \mathrm{keV}$. The $X$-axis refers to time since burst onset in sec. The horizontal dashed lines indicate the level of the flux at which the Eddington limit is reached in the main burst.

radiation (in't Zand et al. 2011). Another similar example was reported by Degenaar et al. (2013).

The two bursts are quite similar. They are of intermediate duration, show precursors of similar short duration (32-43 ms), have similar superexpansion durations (1.15-1.25 s), have similar Eddington-limited durations (75 s), and arrive from H-poor UCXBs with low accretion rates onto presumably cool NSs.

\section{The two precursors}

\subsection{Light curves}

Figure 2 provides the full details of both precursor light curves. The data have been collected from four PCUs for 4U 0614+09 and two PCUs for 2S 0918-549. Combined with the larger distance for $2 \mathrm{~S}$ 0918-549, this implies worse statistics for this source.

The following observations can be made from the light curves:

1. the precursors last $43 \mathrm{~ms}(4 \mathrm{U} 0614+09)$ and $32 \mathrm{~ms}(2 \mathrm{~S} 0918$ 549);

2. both burst rises are just resolved and reach the Eddington limit, as determined from the maximum in the main burst phase, very quickly - within about $0.5 \mathrm{~ms}$;

3. the intensities of the bursts surpass the Eddington limit as measured in the main burst (see also Fig. 3) by factors of 2.6 and 3.1, respectively (after correction for dead times in the PCA; see Appendix B);
4. the spectrum softens immediately once the Eddington limit is first reached, within $0.5 \mathrm{~ms}$ from burst onset (as is most easily observed by the increasing $<6 \mathrm{keV}$ intensity and more or less constant $>6 \mathrm{keV}$ intensity);

5. there is considerable variability on submillisecond timescale. For $4 \mathrm{U} 0614+09$, there is still information contained at the maximum time resolution of $122 \mu \mathrm{s}$. It shows a spike $4 \mathrm{~ms}$ into the burst which lasts $2 \mathrm{~ms}$. Later, after $12 \mathrm{~ms}$, it exhibits an unresolved spike which lasts less than $122 \mu \mathrm{s}$. The Poisson probability for the flux to rise so high above the local average in a single trial is only $6.6 \times 10^{-6}$. Coincidental or not, there appear to be three additional spikes at smaller significance: one before and two after the major spike. The most significant one has a chance probability for such a high flux or higher of $2.7 \times 10^{-3}$. The three wait times are exactly $5.0 \mathrm{~ms}$. The spikes arrive from higher energy photons ( $>6 \mathrm{keV}$ ). The data on 2S 0918-549 are of less statistical quality, but they also show ms variability. There is a marked spike immediately at the start of the burst, lasting $1 \mathrm{~ms}$, after which the flux drops by about $75 \%$.

\subsection{Measurement of expansion speed}

We analyzed the evolving spectrum of the precursors and main bursts. For each time bin we extracted the well-calibrated (Jahoda et al. 2006; Shaposhnikov et al. 2012) 2.8-30 keV spectrum (this involves standard-2 channels 3 and onward, counting 
J. J. M. in 't Zand et al.: Relativistic outflow from two thermonuclear shell flashes on neutron stars
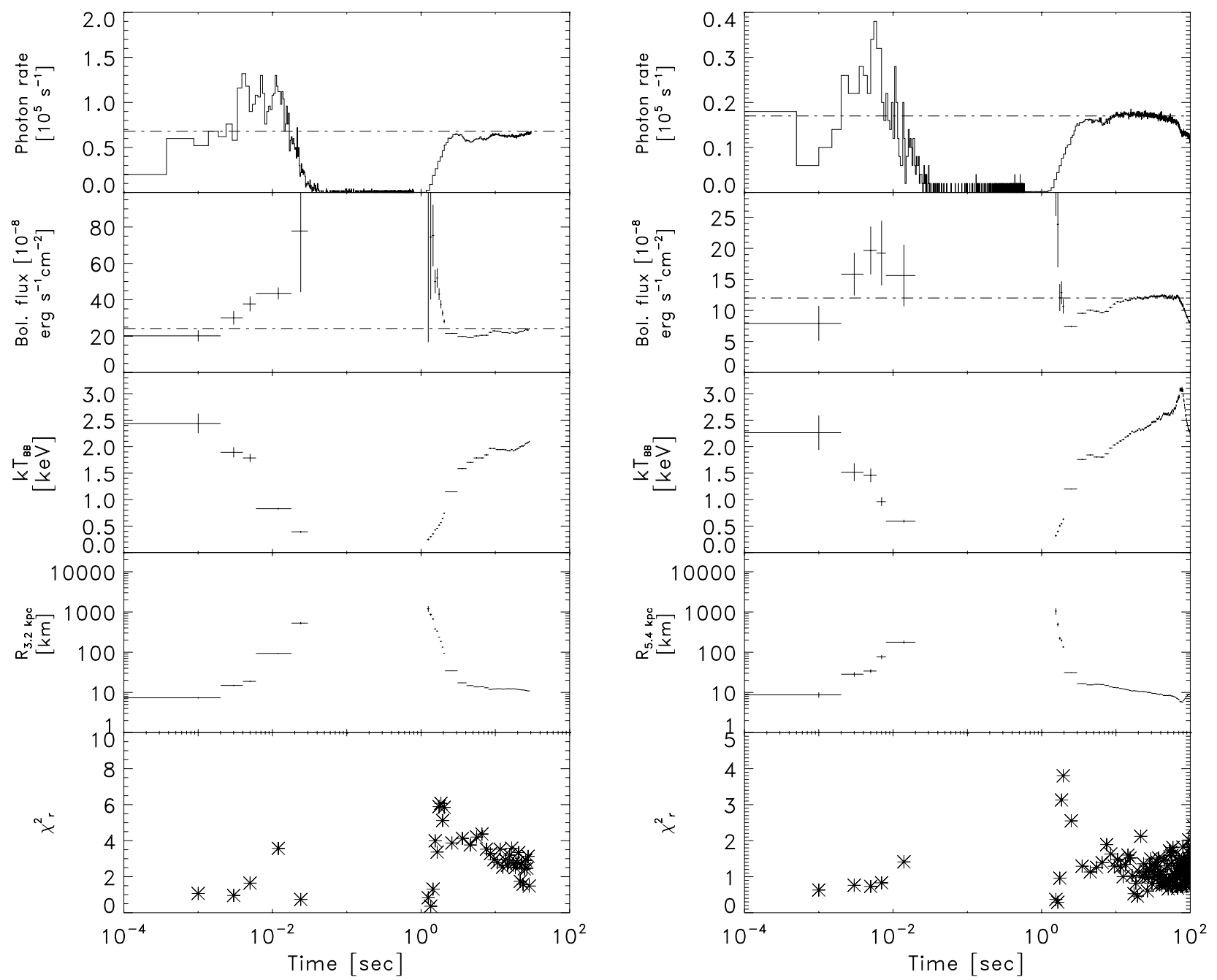

Fig. 3. Time-resolved spectroscopy of 4U 0614+09 (left) and 2S 0918-549 (right). The dash-dotted lines indicate maximum flux levels measured as late as possible in the burst. The spectral model is a simple Planck function. The count rate (top panel) is not corrected for dead time while the bolometric flux and radius are. We note that the Cash statistic was employed for finding the best fit, not the $\chi_{\mathrm{r}}^{2}$ parameter shown in the bottom panels.

from 0). The spectra were modeled with an absorbed Planck function, with $N_{\mathrm{H}}$ fixed to $3 \times 10^{21} \mathrm{~cm}^{-2}$ for both sources (Juett et al. 2001), which is actually a negligible amount in the $2.8-30 \mathrm{keV}$ bandpass. The blackbody temperature and normalization were fitted using the software package XSPEC version 12.8.1g (e.g., Arnaud 1996). The number of photons is sometimes small and we decided to employ the Cash statistic (Cash 1979) to search for the best-fit parameter values. We ignored the cosmic and accretion background since their contribution is negligible (a few tenths of a percent). We note that the $\mathrm{X}$-ray radiating part of the accretion disk is blocked out by the expanding photosphere (e.g., Molkov et al. 2000; in't Zand \& Weinberg 2010). Figure 3 shows the results. For 4U 0614+09, the temperature drops to a low value of $0.391 \pm 0.018 \mathrm{keV}$ and the radius increases to $935 \pm 193 \mathrm{~km}$ before the signal is lost. This happens within $32 \mathrm{~ms}$ and the radius change translates to an average speed of $(3.9 \pm 0.8) \times 10^{4} \mathrm{~km} \mathrm{~s}^{-1}$ during that time interval. If the evolution of the radius is studied more closely, an acceleration can be seen. The last two radius measurements of the precursor translate to a speed of $(1.1 \pm 0.3) \times 10^{5} \mathrm{~km} \mathrm{~s}^{-1}$ or $0.3 c$.

The apparent speed in $2 \mathrm{~S} 0918-549$ is lower than in $4 \mathrm{U} 0614+09$ by a factor of 3 . The last precursor radius is $242 \pm$ $76 \mathrm{~km}$. With a start value of $10 \mathrm{~km}$, the radius change translates to an average speed of $(1.7 \pm 0.5) \times 10^{4} \mathrm{~km} \mathrm{~s}^{-1}$. The radial change between the last two precursor data points translates to $(2.9 \pm 0.9) \times 10^{4} \mathrm{~km} \mathrm{~s}^{-1}$ or $0.1 c$.

These speed measurements are uncertain. The large radii are measured from the Wien tail of the Planck function; the peak of the Planck function is outside the PCA bandpass. This introduces large uncertainties in derived temperatures and emission areas. However, the conclusion that the speed is a few tenths of the speed of light is justified and supported by the mere fact of the short duration. The PCA loses the signal from a blackbody if it becomes cooler than $0.25 \mathrm{keV}$ (in't Zand \& Weinberg 2010). For a constant Eddington luminosity, the equivalent radius would be larger than $10^{3} \mathrm{~km}$. If this happens within the precursor duration of $\approx 30 \mathrm{~ms}$, the average speed must be $0.1 \mathrm{c}$. The data clearly point to mildly relativistic outflows.

A caveat that is generally encountered in the analysis of burst spectra is that they are expected to deviate from the Planck function because of inverse Compton scattering in the NS atmosphere. This introduces a systematic difference between the measured so-called color temperature and the actual effective temperature. Theoretical work (e.g., London et al. 1986; Pavlov et al. 1991; Suleimanov et al. 2012) suggests that the effective temperature is always lower than the color temperature. To arrive at the same flux, the emission area needs to be larger by 
approximately the same factor squared. This implies that our velocities actually correspond to lower limits.

Although the errors are large, the bolometric flux during the precursors is seen to increase above the Eddington limit as measured in the main burst phase after the superexpansion. The bolometric flux during the precursor of $4 \mathrm{U} 0614+09$ is seen to peak at $1.83 \pm 0.13$ times the level seen during the main phase. For $2 \mathrm{~S} 0918-549$ this is $1.63 \pm 0.27$. These values compare to 3.1 and 2.6, respectively, found in Sect. 3 which apply to the PCA photon count rate instead of the bolometric flux.

The spectral analysis of the precursor of 4U 0614+09 was repeated by matching the time bins to the most important features of the light curve. Particularly, we defined time bins that match the flares at 4 and $12 \mathrm{~ms}$. We find that the flare at $4 \mathrm{~ms}$ is hotter than the trend (1.8 versus $1.4 \mathrm{keV}$ at about $3 \sigma$ significance), while the flare at $12 \mathrm{~ms}$ does follow the trend. The temperature accuracies for both flares are similar.

We note that the spectral analysis is incomplete for the main burst phase. In contrast to the situation during the precursor phase, the X-ray radiating part of the accretion disk is thought to be visible again during the main burst phase and interferes slightly with the burst spectrum. We do not pursue a full analysis here because we are interested predominantly in the precursor. For a more complete analysis of the main burst phase, the reader is referred to in't Zand \& Weinberg (2010) and in't Zand et al. (2011). See also in't Zand et al. (2013) and Worpel et al. (2013) for a more extensive study of the behavior of the accretion disk spectrum during bursts.

\section{Discussion}

Our precursor analysis shows three peculiarities: short precursor durations, super-Eddington fluxes and short rise times. These observations point to exceptional conditions. From the following discussion we conclude that the short rise time measured for the two bursts is consistent with the helium burning of a thick layer $\left(y_{\text {ign }} \gtrsim 10^{10} \mathrm{~g} \mathrm{~cm}^{-2}\right)$, in which temperatures rise high enough to initiate the ${ }^{12} \mathrm{C}(p, \gamma){ }^{13} \mathrm{~N}(\alpha, p){ }^{16} \mathrm{O}$ bypass so that probably a detonation is invoked that allows for a fast spreading of the flame which is unaffected by NS rotation, and a fast ejection of a shell. Radial velocities are mildly relativistic.

\subsection{Short precursor time and super-Eddington fluxes: relativistic outflow}

The precursors we observe are the shortest for any thermonuclear flash. For the 36 other precursor bursts listed in Appendix A (excluding one superburst), the durations range between $150 \mathrm{~ms}$ and $4 \mathrm{~s}$. The precursor phenomenon is attributed to photospheric expansion to radii of $10^{3} \mathrm{~km}$ and beyond (Tawara et al. 1984a,b; Lewin et al. 1984). The time-resolved spectroscopy of our bursts (Fig. 3) is consistent with that explanation. Our precursor durations point to an average speed of $10^{3} \mathrm{~km} / t_{\text {prec }} \approx 0.1 c$. A close look at the time-resolved spectroscopy suggests that the speed is not constant and accelerates to $0.3 c$ for $4 \mathrm{U} 0614+09$. This is a mildly relativistic outflow.

The relativistic character of the outflow allows for a natural explanation of the second peculiarity, that of super-Eddington fluxes, provided that the outflow is bulk motion and not, for instance, an optical depth effect. The visible approaching side of the shell will be Doppler boosted by a factor $B$ for the energy flux of

$B=D^{3+\alpha}$ with

$D=1 / \Gamma(1-\beta \cos \theta)$,

$\Gamma$ the Lorentz factor $1 / \sqrt{1-\beta^{2}}, \beta=v / c, \theta$ the angle between the velocity and the line of sight and $\alpha$ the energy spectral index. For a Doppler boost factor equal to the measured super-Eddington ratio $1.7 \pm 0.2$ (for the bolometric luminosity for both sources, see Fig. 3), $\theta=0$, and $\alpha=0, \beta$ would be $0.18 \pm 0.04$. This is similar in magnitude to the estimate from the time-resolved spectroscopy. It is worth noting that expansion speeds of a few tenths of the speed of light are of the same magnitude as the escape velocity from the NS surface $(0.6 c$ for a canonical NS).

Apart from this special relativistic effect, one expects general relativistic effects as well. The Eddington limit as seen by a distant observer depends on the photospheric location in the gravitational well according to

$F_{\text {Edd }, \infty} \propto \sqrt{1-\frac{2 G M}{R c^{2}}}$

with $G$ the gravitational constant, $M$ the NS mass and $R$ the distance to the NS center of mass (e.g., Damen et al. 1990). For a canonical NS, the Eddington limit would be $31 \%$ smaller for $R=10 \mathrm{~km}$ than for $R=\infty$. For $M_{\mathrm{NS}}=2 M_{\odot}$, this would even be $56 \%$. These numbers could explain a part of the effect that we see. However, the same effect should also be visible in other precursor bursts irrespective of the precursor duration, since it only depends on $M$ and $R$. We checked this in the literature, see the references in in't Zand \& Weinberg (2010) and the light curves in in't Zand et al. (2012), and found it not to be the case. Furthermore, it should already be clearly visible in bursts with moderate photospheric expansion. The difference in the Eddington limit between $R=10$ and $R=20 \mathrm{~km}$ should already be $17 \%$. This has not been detected (Damen et al. 1990). General Relativity effects do not seem to be a dominant feature in measurements of $L$. Damen et al. (1990) attribute this to the dominance of systematic effects, such as compositional change in the photosphere, changes in the accretion radiation during the burst and deviations from blackbody radiation and associated errors in bolometric correction. Furthermore, the expected changes in $L$ are smaller than what we observe in our two short precursors.

This would be the first time that Doppler boosting has been detected in a thermonuclear flash, whether a shell flash on a NS or white dwarf, or a Type Ia supernova.

\subsection{Short rise time: flash ignition and flame spreading}

The short duration of the precursors goes hand in hand with short rise times. We find that the rise time to the Eddington limit is $\$ 0.5 \mathrm{~ms}$. The rise time of a burst is expected to be the sum of the nuclear reaction timescale $t_{\text {nuc }}$, the time $t_{\text {wave }}$ it takes the heat wave to travel upward and reach the photosphere, and the time $t_{\text {spread }}$ it takes the flame to spread laterally over the NS surface. The rise time is determined by the longest of these three timescales. We discuss each of these three timescales separately.

\subsection{1. $t_{\text {nuc }}$}

For a helium flash igniting at $y_{\text {ign }} \simeq 10^{10} \mathrm{~g} \mathrm{~cm}^{-2}$ (pressure $P \simeq 10^{24}$ dyne $^{-2}$ for a canonical NS), burning through the $3 \alpha$-process and a chain of $\alpha$-captures yields $t_{\text {nuc }} \simeq 10^{-3} \mathrm{~s}$ (Fujimoto et al. 1981). Weinberg et al. (2006), however, found 


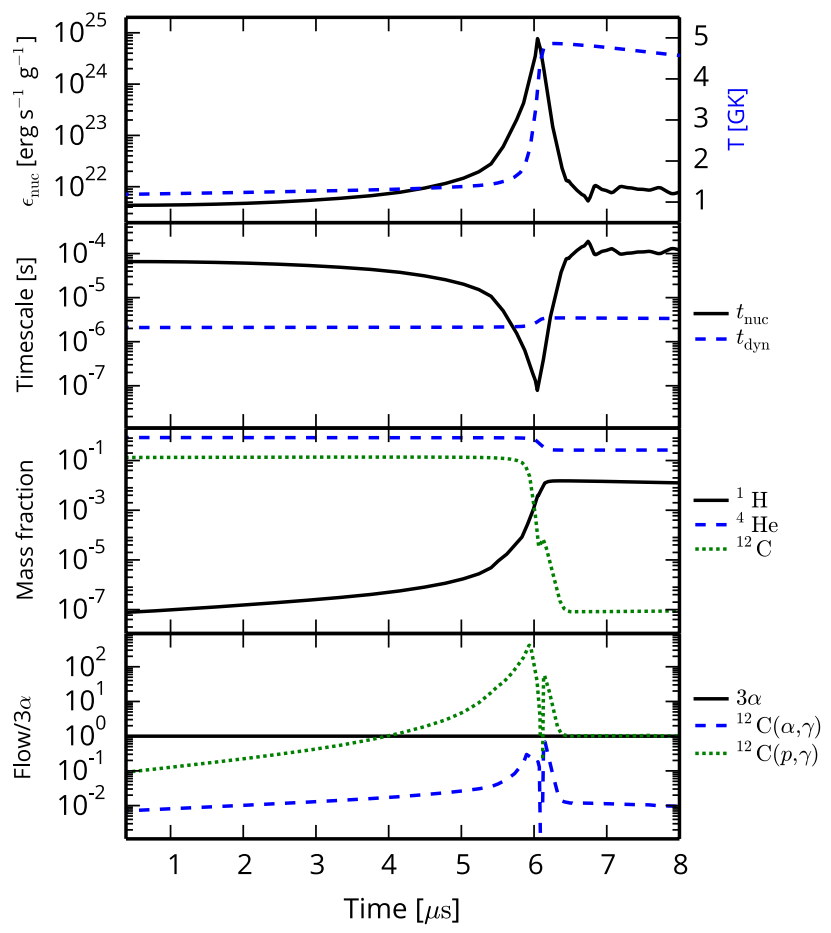

Fig. 4. Details of the nuclear burning at the start of a helium flash, around the time when the specific energy generation rate, $\epsilon_{\text {nuc }}$, is highest. For a KEPLER model in the zone where runaway thermonuclear burning initiates the flash, we show as a function of time, $t$, since the start of the local runaway: $\epsilon_{\text {nuc }}$, temperature $T$ (dashed line), nuclear burning timescale $t_{\text {nuc }}$, dynamical timescale $t_{\text {dyn }}$, mass fractions of three isotopes, and the nuclear flow through several reactions relative to the $3 \alpha$ flow.

for $y_{\text {ign }}=3 \times 10^{8} \mathrm{~g} \mathrm{~cm}^{-2}$ that bypassing the slow ${ }^{12} \mathrm{C}(\alpha, \gamma){ }^{16} \mathrm{O}$ reaction with ${ }^{12} \mathrm{C}(p, \gamma){ }^{13} \mathrm{~N}(\alpha, p){ }^{16} \mathrm{O}$ using protons created in $(\alpha, p)$ reactions substantially reduces $t_{\text {nuc }}$. One-dimensional simulations performed by Wallace et al. (1982) with the hydrodynamic stellar evolution code KEPLER (Weaver et al. 1978) of helium flashes with $y_{\text {ign }} \simeq 10^{10} \mathrm{~g} \mathrm{~cm}^{-2}$ included the ${ }^{12} \mathrm{C}(p, \gamma){ }^{13} \mathrm{~N}(\alpha, p){ }^{16} \mathrm{O}$ reactions. Although Wallace et al. (1982) omitted the production of protons by $(\alpha, p)$ reactions, the importance of this effect became apparent when hydrogen from their accretion composition mixed into the helium burning region, and $t_{\text {nuc }}$ was briefly reduced to below $1 \mu \mathrm{s}$. To further investigate this effect, we performed new KEPLER simulations of the accretion of $98 \%$ by mass ${ }^{4} \mathrm{He}$ and $2 \%{ }^{14} \mathrm{~N}$ onto a canonical NS. We used a modern version of KEPLER that was used in recent X-ray burst models, and we refer to the respective papers for details (Woosley et al. 2004; Heger et al. 2007a,b; Keek \& Heger 2011). Nuclear burning is implemented using a large adaptive network with thermonuclear rates from a compilation by Rauscher et al. (2003), which includes the $3 \alpha$ reaction, $\alpha$ - and $p$-capture reactions, as well as $(\alpha, p)$ reactions, among others. For our study, we select a burst that ignites close to the bottom of the accreted fuel column at $y_{\text {acc }}=1.7 \times 10^{10} \mathrm{~g} \mathrm{~cm}^{-2}$, which is on the order of the values inferred for the bursts discussed in this paper.

At the burst onset, heating by $3 \alpha$ burning creates a convection region around $y_{\text {ign }}$, and local runaway burning starts after $16 \mathrm{~s}$ in one zone at $y_{\text {ign. }}$. We study the burning processes in this zone, as we expect $t_{\text {nuc }}$ to be shortest here (Fig. 4). The density in this zone is $\rho_{\text {ign }}=2.3 \times 10^{7} \mathrm{~g} \mathrm{~cm}^{-3}$. Nuclear burning before the local runaway increases the temperature to $T \simeq 1 \mathrm{GK}$, and $(\alpha, p)$ reactions produce a small number of protons. Even though this number is only $4 \times 10^{-7}$ times the number of $\alpha$ particles, the ${ }^{12} \mathrm{C}(p, \gamma)$ reaction already has a much higher rate than ${ }^{12} \mathrm{C}(\alpha, \gamma)$. The reaction ${ }^{12} \mathrm{C}(p, \gamma){ }^{13} \mathrm{~N}(\alpha, p){ }^{16} \mathrm{O}$ bypasses the slower reaction ${ }^{12} \mathrm{C}(\alpha, \gamma){ }^{16} \mathrm{O}$, and provides more seed nuclei for $\alpha$-capture and $(\alpha, p)$ reactions. The latter reaction further increases the proton mass fraction to as much as $1.5 \%$. At the peak of the specific energy generation rate, $\epsilon_{\text {nuc }}$, the nuclear flow through ${ }^{12} \mathrm{C}(p, \gamma)$ exceeds the $3 \alpha$ flow by several orders of magnitude. Most of the ${ }^{12} \mathrm{C}$ is destroyed, providing seed nuclei for further fast $\alpha$-capture reactions. As the temperature rapidly increases to $5 \mathrm{GK}$, there is a brief dip in the flows of ${ }^{12} \mathrm{C}(p, \gamma)$ and ${ }^{12} \mathrm{C}(\alpha, \gamma)$, because photo disintegration enhances the rate of the reverse reactions. Subsequently, nuclear burning reaches ${ }^{56} \mathrm{Ni}$, which on a longer timescale undergoes electron capture to form ${ }^{56} \mathrm{Fe}$. This is the most abundant isotope in the ashes. Finally, the flow through ${ }^{12} \mathrm{C}(p, \gamma)$ equals the $3 \alpha$ flow, as almost all carbon created by the latter reaction is immediately destroyed by the former.

We estimate $t_{\text {nuc }}$ from the ratio of the specific internal energy and $\epsilon_{\text {nuc }}$. At the peak of $\epsilon_{\text {nuc }}$, it reaches a minimum of $t_{\text {nuc }}=$ $7.8 \times 10^{-8} \mathrm{~s}$. Apart from the conclusion that it is well below our measured rise times, it has the important implication that this is shorter than the dynamical timescale of $t_{\mathrm{dyn}} \simeq 3 \mu \mathrm{s}$ (defined as the ratio of the pressure scale height and the sound speed). In that zone, $t_{\text {nuc }}$ is shorter than $t_{\text {dyn }}$ for $0.49 \mu$ s and within this short time the helium mass fraction is reduced to 0.26 , while the burning generates $0.92 \times 10^{18} \mathrm{erg} \mathrm{g}^{-1}$.

\subsection{2. $t_{\text {wave }}$}

Without the ${ }^{12} \mathrm{C}(p, \gamma){ }^{13} \mathrm{~N}(\alpha, p)^{16} \mathrm{O}$ bypass, burning is subsonic for helium bursts with $y_{\text {ign }} \simeq 10^{10} \mathrm{~g} \mathrm{~cm}^{-2}$. Burning spreads from the ignition depth to lower depths as a deflagration, and heat is transported towards the surface by convection on a timescale of $10^{-5}$ s. Previous models with $y_{\text {ign }}=3 \times 10^{8} \mathrm{~g} \mathrm{~cm}^{-2}$ (including the bypass reaction; Weinberg et al. 2006) found that convection stalls at a depth where the thermal timescale is $\sim 1 \mathrm{~ms}$, which set the burst rise time. For $y_{\text {ign }} \simeq 10^{10} \mathrm{~g} \mathrm{~cm}^{-2}$ convection likely reaches even closer to the photosphere, producing a shorter rise.

Our KEPLER model that includes the ${ }^{12} \mathrm{C}(p, \gamma){ }^{13} \mathrm{~N}(\alpha, p){ }^{16} \mathrm{O}$ bypass, however, has hydrodynamic burning, when $t_{\text {nuc }}<t_{\text {dyn }}$. For one-dimensional (Wallace et al. 1982) and two-dimensional (Zingale et al. 2001) models that satisfy this condition, the flame spreads as a detonation, and launches a shock towards the surface. We note that these models require a larger $y_{\text {ign }}$, as they lack the enhanced energy generation rate due to the ${ }^{12} \mathrm{C}(p, \gamma){ }^{13} \mathrm{~N}(\alpha, p){ }^{16} \mathrm{O}$ bypass. The KEPLER model also includes a shock that reaches the surface and a shock-breakout peak in the light curve with a timescale of $\lesssim 10^{-6}$ s. Fall-back of the shocked outer atmosphere on a dynamical timescale of $t_{\text {dyn }} \simeq$ $3 \times 10^{-6} \mathrm{~s}$ heats the atmosphere, which leads to a fast rise of the light curve. Although the precise details of the shock breakout are likely not accurately reproduced in our one-dimensional simulation (see, e.g., Keek \& Heger 2011), the heating of the photosphere on $t_{\text {dyn }}$ is robust.

One-dimensional models may not be best suited to determine the presence of detonation. Detonation requires not only $t_{\text {nuc }}<t_{\text {dyn }}$, but also adherence to the Zel'dovich criterion (e.g., Zel'Dovich et al. 1970) which states that the initial spontaneously supersonic burning region should be large enough that geometric dilution does not prematurely terminate the detonation. Weinberg \& Bildsten (2007) apply the Zel'dovich criterion to the case of carbon flashes, but show that uncertainties remain large and prevent a definite determination. However, irrespective 
of whether convective or shock heating takes place, both produce timescales $t_{\text {wave }}$ shorter than the observed rise times of our two bursts.

\subsection{3. $t_{\text {spread }}$}

Models proposed for the lateral propagation of the flame fall into two categories: detonations and deflagrations. In the case of detonations, after the ignition has started at some location, the flame starts a shock wave which, advancing, ignites the rest of the fluid via compression (in different possible ways, see Zingale et al. 2001; Simonenko et al. 2012). The flame proceeds at the same speed as the shock and can reach supersonic speeds on the order of $10^{9} \mathrm{~cm} \mathrm{~s}^{-1}$. Detonations are typically revealed in numerical simulations of deep helium ignition $\left(y_{\mathrm{igm}} \gtrsim 4 \times 10^{11} \mathrm{~g} \mathrm{~cm}^{-2}\right.$; Zingale et al. 2001; Simonenko et al. 2012), but so far the rotation of the star and the effects of the Coriolis force have been neglected. Such effects may prevent a large bulk motion (Cavecchi et al. 2013).

On the other hand, deflagrations are driven by thermal conduction (Fryxell \& Woosley 1982; Cavecchi et al. 2013) and proceed more slowly. Even if hydrodynamics, via the Coriolis force, can increase the speed due to geometrical effects (Spitkovsky et al. 2002; Cavecchi et al. 2013), velocities remain lower than $\sim 10^{6} \mathrm{~cm} \mathrm{~s}^{-1}$ (Cavecchi et al. 2013) in the regime of ordinary bursts, implying that the NS surface is completely covered only after roughly $t_{\text {spread }}=1 \mathrm{~s}$.

Our bursts take place at a column depth of $y \sim 10^{10} \mathrm{~g} \mathrm{~cm}^{-2}$, intermediate between the regimes explored so far. Our data suggests that the flame spreads over half the NS circumference in $t_{\text {spread }} \lesssim 1 \mathrm{~ms}$. The flame spread time appears to be out of the realm of deflagrations and in that of detonations. A tentative conclusion is that we are dealing with detonations.

However, measurements in a third burster show that this is not so straightforward. Short rise times also occasionally appear in bursts with lower ignition depths. Some superexpansion bursts from $4 \mathrm{U} 1820-30$ have precursors lasting only $0.15 \mathrm{~s}$ with rise times of $30 \mathrm{~ms}$ (see Appendix A). These bursts are short, are most likely due to pure helium ignition (Cumming 2003), have e-folding decay times less than $10 \mathrm{~s}$ and should, therefore, have ignition column depths on the order of $10^{8} \mathrm{~g} \mathrm{~cm}^{-2}$. Therefore, ignition depth is not the only parameter important for whether the flame propagates as a deflagration or detonation. Could the NS spin rate be the other one? The tentative measurement of a spin of $415 \mathrm{~Hz}$ for $4 \mathrm{U}$ 0614+09 (Strohmayer et al. 2008) suggests it is not. The Coriolis force will be strong, but apparently it is not an issue. This may be explained by the absence of bulk motion, for instance a flame propagating as a pressure wave in a detonation.

Perhaps we are dealing with neither deflagration nor detonation. In the previous section we discuss a simulation showing an extended convection zone for $16 \mathrm{~s}$ at burst onset. The convection is responsible for the heat transport up to a certain height, creating a much shallower radial temperature distribution that is near the ignition condition. The lateral temperature distribution is expected to be closer to uniform than the radial distribution (e.g. Weinberg \& Bildsten 2007), so that the fuel layer may be critically close to ignition throughout. It may not take much lateral heat transport to ignite neighboring fuel pockets and the ignition may quickly spread over the NS. This is not a detonation, but it may have similar spread velocities (this so-called auto- or selfignition is discussed for chemical combustibles in, e.g., Frolov et al. 1992; Makhviladze \& Rogatykh 1991; Bartenev \& Gelfand 1990). In this scenario, the distinguishing factor of bursts with fast rises from those with slow rises would be the strong lateral homogeneity in the temperature distribution.

\subsection{The peculiarity of these two bursts}

The question arises why particularly these two bursts have such short precursors. Apart from this characteristic, the bursts do not seem to be exceptional. The variety of other bursts with precursors is large, without a clear trend. They include short and long bursts ( $\lesssim 1 \mathrm{~min}$ to $1 \mathrm{~h}$ ) and precursors ranging between $0.15 \mathrm{~s}$ and $4 \mathrm{~s}$, with no correlation between them. All bursts with precursors appear to arrive from hydrogen-deficient UCXBs (in't Zand \& Weinberg 2010; in't Zand et al. 2012). Therefore, while the deficiency of hydrogen seems to be a prerequisite for photospheric expansion strong enough for precursor appearance, the rapidity of the precursor must be determined by other parameters. We propose that the helium abundance is an important such parameter. In 4 U 1820-30, the abundance is high enough (Cumming 2003) to generate the needed power for a fairly fast $(0.15 \mathrm{~s})$ expansion $\left(v=\sim 10^{3} \mathrm{~km} / 0.15 \mathrm{~s}=7 \times 10^{3} \mathrm{~km} \mathrm{~s}^{-1}\right)$, even though the ignition column depth is limited (i.e., on the order of $10^{8} \mathrm{~g} \mathrm{~cm}^{-2}$ ). Perhaps also the high accretion rate in 4U 1820-30 helps. This makes the start value of the NS temperature high, so that temperatures during the flash rise high enough to invoke the fast bypass reaction in the nuclear reaction chain. Perhaps our two cases are exceptional in having a high helium abundance as well as a large ignition column depth. This would provide an exceptionally large luminosity and radiative driving to push the shell to exceptionally high velocities, and invoke convection zones large enough to provide short burst rises. In other long superexpansion bursts (e.g., in 4U 1722-30 and SLX 1735-269; Molkov et al. 2000, 2005, respectively), the helium abundance may be smaller.

The duration of the superexpansion stage (i.e., the time between the onset of the precursor and that of the main burst phase) must be proportional to the initial column thickness of the shell divided by the speed. For a duration of $1.2 \mathrm{~s}$ and a speed of $0.3 c$, the shell distance to the NS is $10^{5} \mathrm{~km}$ when it becomes optically thin, which translates to a dilution factor of $10^{8}$. Therefore, if the speed of the shell is constant at $0.3 c$ (0.1c for 2S 0918-549), the initial shell column thickness would be $10^{8} \mathrm{~g} \mathrm{~cm}^{-2}\left(10^{7} \mathrm{~g} \mathrm{~cm}^{-2}\right)$ which is at the limit of the energy constraint of $10^{-2} y_{\text {ign. }}$. Accurate calculations are outside the scope of this paper and need to take into account the radial structure of the shell (i.e., the geometric thickness) and the speed evolution, both of which depend on continued driving by radiation pressure (the flux remains Eddington-limited for a considerable time after the precursor) and possibly line driving (e.g., in't Zand \& Weinberg 2010).

\section{4. (Sub)millisecond variability}

There is considerable variability in both precursors. In $4 \mathrm{U} 0614+09$, we see after $2.5 \mathrm{~ms}$ a spike that lasts $1.5 \mathrm{~ms}$. Notably, this spike is due to $>6 \mathrm{keV}$ photons only and the timeresolved spectroscopy shows the temperature to temporarily increase. In 2S 0918-549, we see an initial $1 \mathrm{~ms}$ long spike which subsides within another ms. This is visible in both bandpasses, in contrast to the spike in $4 \mathrm{U} 0614+09$, although the statistical quality precludes a strong statement about this. Additionally, $4 \mathrm{U} 0614+09$ shows flares that are so short that they cannot be resolved with the $122 \mu$ s resolution of the data. Interestingly, this is the light-crossing time of a mere $36 \mathrm{~km}$.

The data point to an optically thick outflow. There is no reason to believe that this outflow should be isotropic and with one 
speed. An irregular structure is plausible, with different pockets of gas traveling at different speeds. This may result in collisions that give rise to brief episodes of additional radiation, very much like in the prompt emission of gamma-ray bursts where internal shocks are thought to be responsible for the large variability in the prompt emission (Rees \& Meszaros 1994).

Although our bursts are very bright, no burst oscillations were detected (for a review of burst oscillations, see Watts 2012). For the burst from $4 \mathrm{U} 0614+09$, this is not a meaningful statement because there is only PCA data for the Eddington-limited phase. Never has a burst oscillation been detected during such a phase in any burst. For 2S 0918-549, it is a meaningful statement. A search by in't Zand et al. (2011) for burst oscillations revealed none; the fractional rms upper limit is between $3.9 \%$ and $6.9 \%$ for data stretches of 4 and $1 \mathrm{~s}$, respectively. The lack of burst oscillations in 2S 0918-549 may be related to the fast flame spreading preventing the development of a strong enough anisotropy to give rise to burst oscillations.

\section{Conclusion and future prospect}

We have studied the exceptional onset of two intermediateduration thermonuclear X-ray bursts that provide insight into the physics of flame spreading, nuclear burning and the dynamics of radiatively driven outflows. We find that the absence of hydrogen and the deep ignition of helium may yield detonation-like explosions that quickly traverse the NS radially and laterally, and have large radiative powers that may result in a relativistic outflow. Better understanding of this phenomenon needs to come from additional theoretical work and observations with improved instrumentation.

So far, theoretical work on the outflow focused on the quasistatic stages later on during the Eddington-limited phase (see Sect. 1). It would be useful to extend this to the initial stage that is suspected to be essential for the development of the shell as an alternative explanation of the superexpansion. In addition, a theoretical study would be useful of the development of the structure of the shell, as a means to explain the end stage of superexpansion (i.e., rise times of the main burst phase and the variability that is sometimes seen during this rise). Furthermore, it would be useful to extend the simulations of ignition to include the auto-ignition phase, for instance to be able to quantify the Zel'dovich criterion radially and laterally, and to extend the simulations of flame spreading at intermediate column depths $\left(\sim 10^{10} \mathrm{~g} \mathrm{~cm}^{-2}\right)$, to investigate in particular the initiation of detonations and short rise times as a function of ignition depth, helium abundance, pre-burst temperature, and NS spin rate.

This study shows that X-ray bursts exhibit significant and interesting variability on submillisecond timescales that reveal dynamic and localized phenomena on NS surfaces. The bursts we study here are among the top $0.5 \%$ brightest bursts seen thus far, but still only the largest instrument flown thus far (in the relevant bandpass) enabled the submillisecond study. With an order-ofmagnitude larger photon collecting area in the same bandpass, more bursts can be studied, possibly a few tens, some of which can be studied at improved statistical accuracy. The LOFT mission concept with $10 \mathrm{~m}^{2}$ photon collecting area (Feroci et al. 2012) is excellently suited for these studies. The envisaged Athena mission with $2 \mathrm{~m}^{2}$ collecting area for the L2 ESA opportunity (Nandra et al. 2013) does not have the optimum bandpass (the effective area at $6 \mathrm{keV}$ is expected to be similar as the PCA on RXTE) and the observing program will not have as much emphasis on X-ray bursters, but the soft bandpass will improve the possibility of measuring the soft spectra at large expansion phases and will better constrain the photospheric radius than was possible with the PCA. Furthermore, the higher spectral resolution may reveal narrow spectral features resulting from absorption by the expelled material which may be enriched with nuclear ashes. This may particularly be possible during the initial stages of the main burst when the expelled material becomes optically thin. Unfortunately, it will be at least another 12 years before Athena or LOFT may become operational. In the mean time, ASTROSAT (to be launched within a few years; Agrawal 2006) will provide the best opportunity to continue the study of superexpansion bursts with a similar combined capability as RXTE-PCA (through its LAXPC instrument) and SwiftXRT (through its SXT instrument).

Acknowledgements. We thank Andrew Cumming for calculating the ignition column depth of the burst from 2S 0918-549, Nevin Weinberg and Tullio Bagnoli for useful discussions, and Craig Markwardt from the RXTE Team for advice on PCA data analysis. The authors have been members of International Teams in Space Science on type I X-ray bursts, sponsored by the International Space Science Institute (ISSI) in Bern (Team IDs 190 and 237). They are grateful to ISSI for providing a discussion platform that helped initiate the present work. L.K. acknowledges support from NASA ADAP grant NNX13AI47G and NSF award AST 1008067.

\section{Appendix A: Superexpansion bursts}

Table A.1 presents a list of all superexpansion bursts that we are aware of. This table is an augmented version of Table 1 published by in't Zand \& Weinberg (2010).

The shortest precursor after those presented in this paper was found in a burst from 4U 1820-30, which was published in in't Zand et al. (2012) as burst no. 4 (MJD 54958.740; ObsID 94090-02-02-01). The light curve is shown in Fig. A.1. The rise time is $30 \pm 2 \mathrm{~ms}$. Another higher quality burst is shown in the same figure. This also has a short rise time and a somewhat longer duration. It is burst no. 7 (MJD 54981.187; ObsID 94090-01-05-00).

\section{Appendix B: PCA dead time correction for high time resolution}

The formal recipe for dead time correction ${ }^{2}$ uses $0.125 \mathrm{~s}$ resolution standard- 1 data and is $1 /\left(1-10^{-5} * C 1-1.5 \times 10^{-4} * C 2\right)$ with $C 1$ the combined rate per PCU of the Good Xenon Events, the Propane events and the coincidence (so-called Remaining) Events. The symbol $C 2$ represents the count rate of the Very Large Events (i.e., those that trigger the upper energy discriminator). Unfortunately, this is not useful for the $10^{3}$ times larger time resolution employed here. Therefore, we calculated an alternative dead time correction from only Good Xenon Events, since these dominate the dead time during the bursts, through $1 / 0.98\left(1-1.25 \times 10^{-5} C\right)$ with $C$ the Xenon event rate per PCU from the event mode data. This alternative recipe was calibrated against the formal recipe for a time resolution of $0.125 \mathrm{~s}$ throughout the burst, see Fig. B.1. For 4U 0614+09 at $122 \mu$ s resolution, the dead time fraction rises to $35 \%$ at the peak of the precursor, compared to about $20 \%$ at the peak in the main burst, and for 2 S $0918-549$ to $22 \%$. Without dead time correction, the observed precursor peak count rate is $2.0 \pm 0.1$ times that of the main burst phase, for both bursts. After dead time correction, this rises to $3.1 \pm 0.3$ for $4 \mathrm{U} 0614+09$ and to $2.6 \pm 0.3$ for $2 \mathrm{~S}$ 0918-549.

2 See the RXTE Cook Book at http://heasarc.nasa.gov/docs/ xte/recipes/cook_book.html 
Table A.1. List of 39 bursts with superexpansion.

\begin{tabular}{|c|c|c|c|c|c|}
\hline \multirow[b]{2}{*}{ Instrument/MJD } & \multicolumn{4}{|c|}{ Duration (s) } & \multirow[b]{2}{*}{ Ref. } \\
\hline & $\begin{array}{c}\text { Precursor } \\
\text { phase }\end{array}$ & $\begin{array}{c}\text { Super } \\
\text { expansion } \\
\text { phase } t_{\mathrm{se}}\end{array}$ & $\begin{array}{c}\text { Moderate } \\
\text { expansion } \\
\text { phase } t_{\mathrm{me}}\end{array}$ & $\tau_{\text {decay }}{ }^{a}$ & \\
\hline \multicolumn{6}{|c|}{$4 U 0614+09$} \\
\hline RXTE/51 944.903 & 0.04 & 1.2 & 75 & $40(2)$ & 1 \\
\hline \multicolumn{6}{|c|}{$2 S 0918-549$} \\
\hline RXTE/54 504.127 & 0.03 & 1.2 & 75 & 125 & \\
\hline \multicolumn{6}{|c|}{ A $1246-588$} \\
\hline WFC/50 286.290 & 3.0 & 1.5 & 54 & $38(5)$ & 2 \\
\hline WFC/51539.874 & 1.5 & 6.0 & 25 & $19(1)$ & 2 \\
\hline \multicolumn{6}{|c|}{$4 U$ 1708-23 (probably) } \\
\hline SAS-C/43 181.834 & 4.2 & 6.0 & 304 & $300(50)$ & 3,4 \\
\hline \multicolumn{6}{|c|}{$X B 1715-321$} \\
\hline SAS-C/42 957.620 & 2.4 & 1.5 & 36 & $30^{b}$ & 3 \\
\hline Hakucho/45 170.231 & 3 & 4.0 & 105 & $85(5)$ & 5 \\
\hline \multicolumn{6}{|c|}{$4 U 1722-30$} \\
\hline WFC/50 318.279 & 4.0 & 2.0 & 16 & $18(3)$ & 6 \\
\hline WFC/50 330.196 & 2.0 & 5.0 & 20 & $16(6)$ & 6 \\
\hline WFC/50 348.938 & 3.0 & 3.0 & 15 & $16(5)$ & 6 \\
\hline WFC/50 368.307 & 2.0 & 5.0 & 14 & $19(3)$ & 6 \\
\hline WFC/50 526.311 & 1.0 & 4.0 & 15 & $21(6)$ & 6 \\
\hline WFC/50 536.895 & 2.5 & 3.5 & 11 & $9(3)$ & 6 \\
\hline WFC/50 538.439 & 3.0 & 1.0 & 15 & $19(6)$ & 6 \\
\hline WFC/50 553.130 & 2.5 & 1.5 & 17 & $12(2)$ & 6 \\
\hline WFC/50 892.706 & 3.0 & 2.5 & 18 & $15(2)$ & 6 \\
\hline WFC/50 904.813 & 2.5 & 4.0 & 21 & $19(3)$ & 6 \\
\hline WFC/51 057.579 & 1.5 & 3.0 & 22 & $19(4)$ & 6 \\
\hline WFC/51 231.379 & 2.0 & 5.0 & 17 & $26(11)$ & 6 \\
\hline WFC/51 270.560 & 2.0 & 5.5 & 25 & $14(2)$ & 6 \\
\hline WFC/51 278.690 & 2.5 & 2.5 & 15 & $13(1)$ & 6 \\
\hline WFC/51 422.838 & 2.0 & 6.5 & 22 & $28(5)$ & 6 \\
\hline WFC/51 431.282 & 4.0 & 5.0 & 27 & $26(5)$ & 6 \\
\hline WFC/51 453.377 & 1.5 & 5.5 & 20 & $24(6)$ & 6 \\
\hline WFC/51 461.331 & 4.0 & 3.5 & 19 & $23(4)$ & 6 \\
\hline WFC/51 610.000 & 3.0 & 3.5 & 20 & $17(3)$ & 6 \\
\hline WFC/51 639.966 & 1.5 & 5.0 & 32 & $23(5)$ & 6 \\
\hline WFC/51 956.091 & 2.0 & 3.5 & 41 & $29(10)$ & 6 \\
\hline RXTE/50 395.292 & 3.6 & 1.6 & 23 & $30.2(0.1)$ & 7 \\
\hline \multicolumn{6}{|c|}{ SLX 1735-269 } \\
\hline I'GRAL/52 897.733 & 2.0 & 7.0 & 482 & $600(100)$ & 8 \\
\hline \multicolumn{6}{|c|}{$4 U 1820-30$} \\
\hline $\mathrm{RXTE} / 51430.074^{c}$ & 15.0 & 2.3 & 1400 & 2500 & 9 \\
\hline RXTE/54 956.774 & 0.5 & 0.6 & 3.8 & $3.2(1)$ & 10 \\
\hline RXTE/54 958.740 & 0.15 & 1.1 & 3.8 & $3.4(2)$ & 10 \\
\hline RXTE/54 978.321 & 0.15 & 1.3 & 3.8 & $3.7(2)$ & 10 \\
\hline RXTE/54 978.495 & 0.25 & 1.0 & 3.8 & $3.6(2)$ & 10 \\
\hline RXTE/54 981.187 & 0.30 & 0.7 & 3.2 & $3.5(1)$ & 10 \\
\hline RXTE/54 994.534 & 0.60 & 0.7 & 3.2 & $3.1(2)$ & 10 \\
\hline \multicolumn{6}{|c|}{$M 15 X-2$} \\
\hline Ginga/47 454.730 & 1.5 & 5.5 & 88 & 60 & 11 \\
\hline WFC/51 871.593 & 1.5 & 7.5 & 169 & $155(11)$ & 12 \\
\hline
\end{tabular}

Notes. ${ }^{(a)}$ Numbers in parentheses represent $1 \sigma$ uncertainties in the least significant digit(s). ${ }^{(b)}$ This number is rather uncertain due to incomplete coverage of the burst; ${ }^{(c)}$ superburst.

References. 1 - Kuulkers et al. (2009); 2 - in't Zand et al. (2008); 3 - Hoffman et al. (1978); 4 - Lewin et al. (1984); 5 - Tawara et al. (1984a); 6 - Kuulkers et al. (2003); 7 - Molkov et al. (2000); 8 Molkov et al. (2005); 9 - Strohmayer \& Brown (2002); 10 - in't Zand et al. (2012); 11 - van Paradijs et al. (1990); 12 - in't Zand et al. (2007).

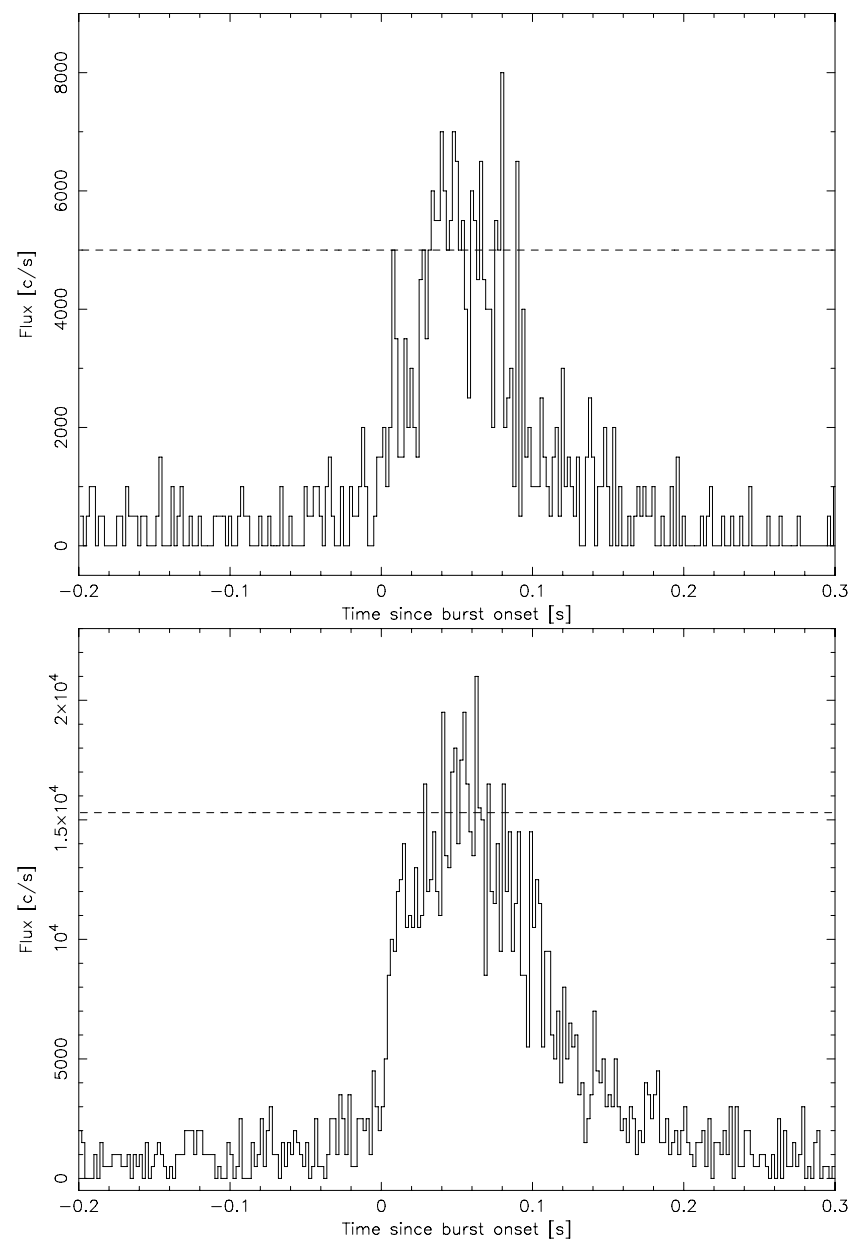

Fig. A.1. Full bandpass light curves of two bursts detected from 4U 1820-30 on MJD 54958.740 (top; one active PCU) and MJD 54981.187 (bottom; three active PCUs). The horizontal dashed lines indicate the Eddington limit in $\mathrm{c} \mathrm{s}^{-1}$ as measured through the peak flux during the main burst phase later on. The time resolution of both light curves is $2 \mathrm{~ms}$.

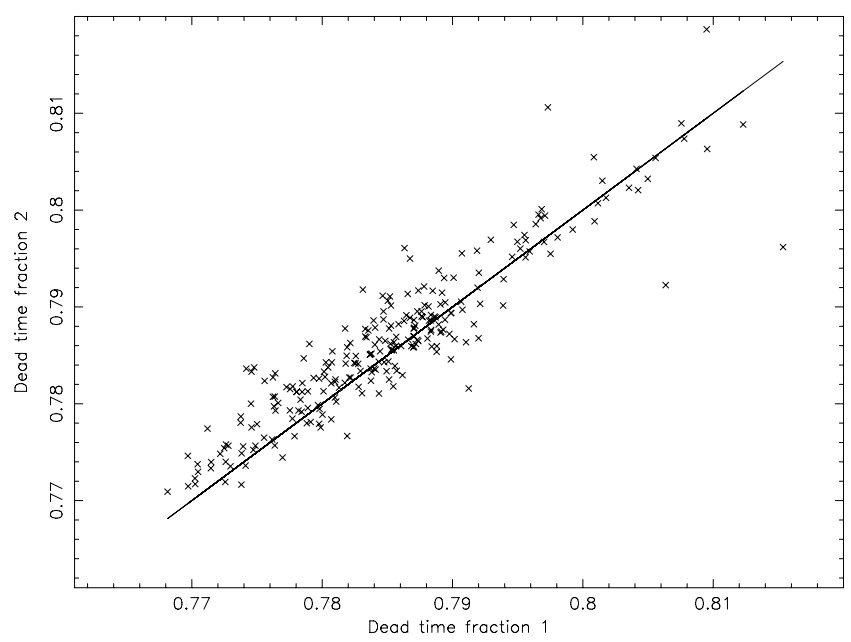

Fig. B.1. Live time fraction 2 calculated from $0.98\left(1-1.25 \times 10^{-5} \mathrm{C}\right)$ against live time fraction 1 calculated according to the formal recipe $1-$ $\left(10^{-5} * C 1+1.5 \times 10^{-4} * C 2\right)$. The line indicates where both are equal.

\section{References}

Agrawal, P. C. 2006, Adv. Space Res., 38, 2989

Arnaud, K. A. 1996, in Astronomical Data Analysis Software and Systems V, eds. G. H. Jacoby, \& J. Barnes, ASP Conf. Ser., 101, 17 
Bartenev, A., \& Gelfand, B. 1990, Progress in Energy and Combustion Science, 26, 29

Belian, R. D., Conner, J. P., \& Evans, W. D. 1972, ApJ, 171, L87

Bildsten, L. 1998, in The many faces of neutron stars, eds. A. Alpar, L. Buccheri, \& J. van Paradijs, NATO ASI (Dordrecht: Kluwer), 419

Brandt, S., Castro-Tirado, A. J., Lund, N., et al. 1992, A\&A, 262, L15

Cash, W. 1979, ApJ, 228, 939

Cavecchi, Y., Watts, A. L., Braithwaite, J., \& Levin, Y. 2013, MNRAS, 434, 3526

Cornelisse, R., Heise, J., Kuulkers, E., Verbunt, F., \& in 't Zand, J. J. M. 2000, A\&A, 357, L21

Cumming, A. 2003, ApJ, 595, 1077

Cumming, A., \& Bildsten, L. 2001, ApJ, 559, L127

Cumming, A., Macbeth, J., in 't Zand, J. J. M., \& Page, D. 2006, ApJ, 646, 429

Damen, E., Magnier, E., Lewin, W. H. G., et al. 1990, A\&A, 237, 103

Degenaar, N., Miller, J. M., Wijnands, R., Altamirano, D., \& Fabian, A. C. 2013 , ApJ, 767, L37

Ebisuzaki, T., Hanawa, T., \& Sugimoto, D. 1983, PASJ, 35, 17

Feroci, M., Stella, L., van der Klis, M., et al. 2012, Exp. Astron., 34, 415

Frolov, S., Gelfand, B., \& Tsyganov, S. 1992, Fizika Goreniya i Vzryva, 28, 13

Fryxell, B. A., \& Woosley, S. E. 1982, ApJ, 261, 332

Fujimoto, M. Y., Hanawa, T., \& Miyaji, S. 1981, ApJ, 247, 267

Fujimoto, M. Y., Sztajno, M., Lewin, W. H. G., \& van Paradijs, J. 1987, ApJ, 319, 902

Galloway, D. K., Muno, M. P., Hartman, J. M., Psaltis, D., \& Chakrabarty, D. 2008, ApJS, 179, 360

Galloway, D., in 't Zand, J., Chenevez, J., Keek, L., \& Brandt, S. 2010, in 38th COSPAR Scientific Assembly, COSPAR Meet., 38, 2445

Giacconi, R., Murray, S., Gursky, H., et al. 1974, ApJS, 27, 37

Grindlay, J., Gursky, H., Schnopper, H., et al. 1976, ApJ, 205, L127

Grindlay, J. E., Marshall, H. L., Hertz, P., et al. 1980, ApJ, 240, L121

Hanawa, T., \& Sugimoto, D. 1982, PASJ, 34, 1

Heger, A., Cumming, A., Galloway, D. K., \& Woosley, S. E. 2007a, ApJ, 671, L141

Heger, A., Cumming, A., \& Woosley, S. E. 2007b, ApJ, 665, 1311

Hoffman, J., Lewin, W., Doty, J., \& et al. 1978, ApJ, 221, L57

in't Zand, J. J. M., \& Weinberg, N. N. 2010, A\&A, 520, A81

in't Zand, J., Verbunt, F., Heise, J., et al. 2004, Nucl. Phys. B Proc. Suppl., 132, 486

in't Zand, J. J. M., Cumming, A., van der Sluys, M. V., Verbunt, F., \& Pols, O. R. 2005, A\&A, 441, 675

in't Zand, J. J. M., Jonker, P. G., \& Markwardt, C. B. 2007, A\&A, 465, 953 in't Zand, J. J. M., Bassa, C. G., Jonker, P. G., et al. 2008, A\&A, 485, 183 in't Zand, J. J. M., Galloway, D. K., \& Ballantyne, D. R. 2011, A\&A, 525, A111 in't Zand, J. J. M., Homan, J., Keek, L., \& Palmer, D. M. 2012, A\&A, 547, A47 in't Zand, J. J. M., Galloway, D. K., Marshall, H. L., et al. 2013, A\&A, 553, A83 in't Zand, J. J. M., Cumming, A., Triemstra, T. L., Mateijsen, R. A. D. A., \& Bagnoli, T. 2014, A\&A, 562, A16

Jager, R., Mels, W. A., Brinkman, A. C., et al. 1997, A\&AS, 125, 557

Jahoda, K., Markwardt, C. B., Radeva, Y., et al. 2006, ApJS, 163, 401

Joss, P. C. 1977 , Nature, 270, 310

Joss, P. C., \& Melia, F. 1987, ApJ, 312, 700

Juett, A. M., Psaltis, D., \& Chakrabarty, D. 2001, ApJ, 560, L59

Keek, L., \& Heger, A. 2011, ApJ, 743, 189
Keek, L., \& in't Zand, J. J. M. 2008, in Proc. of the 7th INTEGRAL Workshop, PoS(Integral08)032

Kuulkers, E., den Hartog, P. R., in 't Zand, J. J. M., et al. 2003, A\&A, 399, 663

Kuulkers, E., in 't Zand, J. J. M., \& Lasota, J.-P. 2009, A\&A, 503, 889

Kuulkers, E., in 't Zand, J. J. M., Atteia, J.-L., et al. 2010, A\&A, 514, A65

Lamb, D. Q., \& Lamb, F. K. 1978, ApJ, 220, 291

Lewin, W. H. G., Vacca, W. D., \& Basinska, E. M. 1984, ApJ, 277, L57

Lewin, W. H. G., van Paradijs, J., \& Taam, R. E. 1993, Space Sci. Rev., 62, 223

London, R. A., Taam, R. E., \& Howard, W. M. 1986, ApJ, 306, 170

Lund, N., Budtz-Jørgensen, C., Westergaard, N. J., et al. 2003, A\&A, 411, L231

Makhviladze, G., \& Rogatykh, D. 1991, Combustion and Flame, 87, 347

Maraschi, L., \& Cavaliere, A. 1977, in X-ray Binaries and Compact Objects, 127

Molkov, S. V., Grebenev, S. A., \& Lutovinov, A. A. 2000, A\&A, 357, L41

Molkov, S., Revnivtsev, M., Lutovinov, A., \& Sunyaev, R. 2005, A\&A, 434, 1069

Nandra, K., Barret, D., Barcons, X., et al. 2013 [arXiv: 1306.2307]

Nelemans, G., Jonker, P. G., Marsh, T. R., \& van der Klis, M. 2004, MNRAS, 348, L7

Nelemans, G., Jonker, P. G., \& Steeghs, D. 2006, MNRAS, 370, 255

Nelson, L. A., Rappaport, S. A., \& Joss, P. C. 1986, ApJ, 304, 231

Nobili, L., Turolla, R., \& Lapidus, I. 1994, ApJ, 433, 276

Paczynski, B., \& Proszynski, M. 1986, ApJ, 302, 519

Pavlov, G. G., Shibanov, I. A., \& Zavlin, V. E. 1991, MNRAS, 253, 193

Rauscher, T., Heger, A., Hoffman, R. D., \& Woosley, S. E. 2003, Nucl. Phys. A, 718,463

Rees, M. J., \& Meszaros, P. 1994, ApJ, 430, L93

Shahbaz, T., Watson, C. A., Zurita, C., Villaver, E., \& Hernandez-Peralta, H. 2008, PASP, 120, 848

Shaposhnikov, N., Jahoda, K., Markwardt, C., Swank, J., \& Strohmayer, T. 2012, ApJ, 757, 159

Simonenko, V. A., Gryaznykh, D. A., Litvinenko, I. A., Lykov, V. A., \& Shushlebin, A. N. 2012, Astron. Lett., 38, 305

Spitkovsky, A., Levin, Y., \& Ushomirsky, G. 2002, ApJ, 566, 1018

Strohmayer, T., \& Bildsten, L. 2006, in Compact stellar X-ray sources (CUP), 113

Strohmayer, T. E., \& Brown, E. F. 2002, ApJ, 566, 1045

Strohmayer, T. E., Markwardt, C. B., \& Kuulkers, E. 2008, ApJ, 672, L37

Suleimanov, V., Poutanen, J., \& Werner, K. 2012, A\&A, 545, A120

Swank, J. H., Becker, R. H., Boldt, E. A., et al. 1977, ApJ, 212, L73

Tawara, Y., Hayakawa, S., \& Kii, T. 1984a, PASJ, 36, 845

Tawara, Y., Kii, T., Hayakawa, S., et al. 1984b, ApJ, 276, L41

van Paradijs, J., Dotani, T., Tanaka, Y., \& Tsuru, T. 1990, PASJ, 42, 633

Wallace, R. K., Woosley, S. E., \& Weaver, T. A. 1982, ApJ, 258, 696

Watts, A. L. 2012, ARA\&A, 50, 609

Weaver, T. A., Zimmerman, G. B., \& Woosley, S. E. 1978, ApJ, 225, 1021

Weinberg, N. N., \& Bildsten, L. 2007, ApJ, 670, 1291

Weinberg, N. N., Bildsten, L., \& Schatz, H. 2006, ApJ, 639, 1018

Woosley, S. E., \& Taam, R. E. 1976, Nature, 263, 101

Woosley, S. E., Heger, A., Cumming, A., et al. 2004, ApJS, 151, 75

Worpel, H., Galloway, D. K., \& Price, D. J. 2013, ApJ, 772, 94

Zel'Dovich, Y. B., Librovich, V. B., Makhviladze, G. M., \& Sivashinskil, G. I. 1970, J. Appl. Mech. Techn. Phys., 11, 264

Zhong, J., \& Wang, Z. 2011, ApJ, 729, 8

Zingale, M., Timmes, F. X., Fryxell, B., et al. 2001, ApJS, 133, 195 